\title{
The role of eye movements in manual responses to social and nonsocial cues
}

\author{
Claudia Bonmassar ${ }^{1} \cdot$ Francesco Pavani $^{1,2,3} \cdot$ Wieske van Zoest $^{1,4}$ \\ Published online: 8 February 2019 \\ (C) The Psychonomic Society, Inc. 2019
}

\begin{abstract}
Gaze and arrow cues cause covert attention shifts even when they are uninformative. Nonetheless, it is unclear to what extent oculomotor behavior influences manual responses to social and nonsocial stimuli. In two experiments, we tracked the gaze of participants during the cueing task with nonpredictive gaze and arrow cues. In Experiment 1, the discrimination task was easy and eye movements were not necessary, whereas in Experiment 2 they were instrumental in identifying the target. Validity effects on manual response time (RT) were similar for the two cues in Experiment 1 and in Experiment 2, though in the presence of eye movements observers were overall slower to respond to the arrow cue compared with the gaze cue. Cue direction had an effect on saccadic performance before the discrimination was presented and throughout the duration of the trial. Furthermore, we found evidence of a distinct impact of the type of cue on diverse oculomotor components. While saccade latencies were affected by the type of cue, both before and after the target onset, saccade landing positions were not. Critically, the manual validity effect was predicted by the landing position of the initial eye movement. This work suggests that the relationship between eye movements and attention is not straightforward. In the presence of overt selection, saccade latency related to the overall speed of manual response, while eye movements landing position was closely related to manual performance in response to different cues.
\end{abstract}

Keywords Attention · Eye movements and visual attention · Face perception

Orienting spatial attention in response to head turning and eye moving is part and parcel of living in a society. Humans are sensitive to the processing of eye gaze, a preference that is thought to be innate and find its roots in evolution (see, e.g., Hood, Willen, \& Driver, 1998). In the course of our lives, we learn to orient attention to more abstract cues like arrows,

Prior dissemination of the data:

- European Conference on Eye Movements, Wuppertal (Germany). August 20-24, 2017. Poster presentation.

- Cognitive Science Arena, Bressanone (Italy). February 23-24, 2018. Talk.

- International Meeting of the Psychonomic Society, Amsterdam (The Netherlands). May 10-12, 2018. Poster presentation.

Claudia Bonmassar

1 Center for Mind/Brain Sciences (CIMeC), University of Trento, Rovereto, Italy

2 Integrative Multisensory Perception Action and Cognition team (ImpAct), Centre de Recherche en Neurosciences de Lyon (CRNL), Lyon, France

3 Department of Psychology and Cognitive Sciences, University of Trento, Trento, Italy

4 School of Psychology, University of Birmingham, Birmingham, UK given that they also convey useful spatial information. We are able to shift our attention according to directions conveyed through signs (e.g., when looking for the right exit from the motorway). Eye gaze or arrows are known as central cuesthat is, stimuli that are presented at the center of the visual field and that enable orientation of attention to another location in space. They differ from peripheral cues, which can capture attention to their location because of an abrupt onset of illumination changes (Posner, 1980).

Many studies have been conducted to understand the characteristics of attention orienting to central cues, mainly focusing on the covert component of it (i.e., the orienting of visuospatial attention without observable eye and body movements; Driver et al., 1999; Friesen \& Kingstone, 1998; Posner, 1978, 1980). The aim of the present work is to investigate the role of overt shifts of attention in the processing of central arrow and gaze cues.

\section{Covert responses to central cues}

Attention orienting to central cues has been extensively investigated by means of the spatial cueing paradigm (Posner, 
1978, 1980). Typically, participants are presented with a central arrow, pointing to the left or to the right, and are asked to respond to a target appearing at previously cued or uncued locations. Manual response times (RTs) are faster for targets appearing at cued locations relative to those appearing at uncued locations. Crucially, in a high proportion of trials, the central cue correctly indicated where the target would appear, creating an incentive for observers to pay attention to the direction of the cue (Jonides, 1981; Kröse \& Julesz, 1989; Müller \& Humphreys, 1991). It has been argued that voluntary goal-driven processes are primarily responsible for the observed behavioral effect. However, more recent studies have challenged the idea that central cues instigate goaldriven orienting of attention only (Corbetta \& Shulman, 2002; Egeth \& Yantis, 1997; Müller \& Rabbitt, 1989). As a matter of fact, centrally presented arrow cues have been found to trigger automatic covert shifts of spatial attention when they are nonpredictive, or even counterpredictive, of the upcoming target location (Friesen, Ristic, \& Kingstone, 2004; Hommel, Pratt, Colzato, \& Godijn, 2001; Ristic, Friesen, \& Kingstone, 2002; Tipples, 2002).

The discovery that central arrow cues are capable of directing attention automatically was in part motivated by the findings from the gaze-cue literature (e.g., Driver et al., 1999; Friesen \& Kingstone, 1998; Friesen et al., 2004; Hietanen, 1999; Hommel et al., 2001; Langton \& Bruce, 1999; Ristic et al., 2002; Ristic \& Kingstone, 2009; Ristic, Wright, \& Kingstone, 2007). In Friesen and Kingstone's (1998) study, participants performed a spatial cueing task with a central schematic face whose gaze direction was nonpredictive of target location. Responses were facilitated by a valid gaze cue compared with a neutral or an invalid one. The attentional benefit was observed for short cuetarget stimulus onset asynchronies (SOAs), adding evidence in favor of a reflexive orienting in response to the eye gaze. However, the aforementioned studies focused on aspects related to covert attention to nonpredictive central cues. Covert measures such as manual RTs depend on inferences regarding the deployment of attention in space. For example, with respect to RTs, the assumption is that attention shifts in line with the direction indicated by the gaze or arrow cue. However, only once the manual response is triggered does it become evident how visual information processing was affected by the direction of the central cues. In contrast to covert mechanisms, which give little information about the underlying temporal dynamics, overt mechanisms of orienting are associated with detectable eye and body movements (Frischen, Bayliss, \& Tipper, 2007; Wright \& Ward, 2008) and provide a window into the ongoing and underlying selection processes. For example, in the central cueing paradigm, concurrent recording of eye movements can yield a measure of eye position at each millisecond along the way, from the moment the cue is presented until the final manual response. In this way, eye movements can provide a way to investigate how overt processing contributes to the manual response to nonpredictive central cues.

\section{Overt responses to central cues}

There are a number of studies that have investigated eye movements in central gaze-cueing (see, e.g., Dalmaso et al., 2015; Kuhn \& Benson, 2007; Kuhn \& Kingstone, 2009; Kuhn, Tatler, \& Cole, 2009b; Kuhn et al., 2011; Kuhn \& Kingstone, 2009; Mansfield, Farroni, \& Johnson, 2003; Ricciardelli, Bricolo, Aglioti, \& Chelazzi, 2002). However, in the majority of these studies, participants were explicitly instructed to make an eye movement to the target. For example, Kuhn and Kingstone (2009) explored the effect of nonpredictive and counterpredictive gaze and arrow cues on the planning and execution of voluntary saccades. The color of the fixation point indicated the saccadic target location. For instance, when the fixation point changed to green, observers were instructed to make a saccade to the right target location, and this location could be either congruent or incongruent with the central arrow or gaze cue. The dependent measures included saccadic RT to the peripheral target and directional eye-movement errors, where saccadic responses were considered correct if they were directed in the general direction of the target (i.e., to the left or right side of screen). The analysis of latency on correct saccades revealed that congruent trials were faster than incongruent trials, both when the gaze cue was nonpredictive and counterpredictive of saccadic target direction. Moreover, error saccades occurred especially on incongruent trials and were characterized by even shorter latencies compared with correct saccades, supporting the idea that erroneous gaze-following occurred automatically. These findings add further evidence in favor of reflexive orienting to gaze cues and extend findings that originated from the literature on covert orienting of attention. In addition, Kuhn and Kingstone (2009) directly compared counterpredictive gaze and arrow cues and showed that saccade latencies and errors were comparable across gaze and arrow cues. This finding conflicts with previous research on covert attention with counterpredictive cues that found evidence for covert reflexive orienting in response to counterpredictive gaze cues, but not arrow cues (Friesen et al., 2004; however, see Tipples, 2008).

While Kuhn and Kingstone (2009) clearly reveal that overt selection is affected by nonpredictive gaze cues or even counterpredictive gaze and arrow cues, as manual RTs were not measured in this work, it remains unclear to what extent saccadic behavior influences or potentially even guides overt manual responses. Moreover, in the work of Kuhn and colleagues, the measure of overt selection in terms of landing position was very general (i.e., to the left or right) and 
involved saccadic responses given after the target and distractor were presented. Thus, it is unclear how more spontaneous overt selection - without explicit motivation to make an eye movement to the target-is affected by the presentation of the cue. The present work will measure saccadic position throughout the duration of the trial to see how the various events (i.e., presentation of the cue and presentation of the target) directly influence dynamic eye-movement behavior. Thus, rather than explicitly instructing observers to make eye movements to a given target, or at a certain point in time, we measured natural variability in oculomotor performance throughout the trial to find out how various oculomotor measures relate to attentional performance.

The goal of the present study is twofold. First, our goal was to investigate the role of spontaneous eye movements in central cueing and to understand whether and how the contingency between eye movements and attention modulates gaze-cue and arrow-cue processing. Second, because we presented participants with both the arrow and the gaze cue, we could investigate potential differences in the processing of uninformative gaze and arrow cues. Eye movements, because they are typically elicited faster than manual responses, represent a more direct measure to probe automatic processing. If gaze cues cause more reflexive orienting than do arrow cues, then we expect to see a greater influence of gaze cues on eyemovement performance compared with arrow cues. For the gaze cue, we used an avatar face stimulus, while the arrow was a simple line drawing. Though our stimuli are vastly different in terms of complexity, validity was manipulated orthogonal to stimulus complexity and not confounded.

We conducted two experiments. Experiment 1 was more exploratory, whereas Experiment 2 was used to confirm the main findings on Experiment 1. In Experiment 1, the discrimination task was relatively easy and could be completed maintaining fixation at the center of the screen. While overt movements were neither required nor necessary to perform the task, making supporting eye movements was not prohibited. Experiment 1 was presented as a typical standard covert attention cueing task and, with regard to eye movements, at the beginning of each main part of the experiment, and after recalibrations, we instructed participants to try and maintain fixation throughout the experiment and respond as fast and as accurately as possible to the target. However, fixation was not enforced, and no feedback was provided on eye-movement behavior. Thus, even though we told observers to maintain fixation, we expected to observe natural variability between trials and across participants in the ability to do so. In a second experiment, the discrimination task was made more difficult, such that eye movements were more critical to performance compared with Experiment 1. The instructions given to participants in Experiment 2 were identical to those of Experiment 1 to ensure that the initial motivation and strategies in terms of fixation were similar across experiments. If eye movements are instrumental in the manual cueing effect, then we expect to find a relationship between eye-movement performance and RT. Moreover, it was predicted that this relationship should be stronger in Experiment 2, where eye movements were critical to performance, compared with Experiment 1, where eye movements were not.

Note that we did not force fixation. Designs with forced viewing conditions are likely to lead to design-specific strategies that would confound our measure of interest, the natural eye-movement behavior. While there are studies that directly compared covert with overt attention independently, the strength of our study is that we studied these concurrently, rather than separately. The present work combines covert and overt measures (i.e., manual RT and eye-movement components) to see how eye movements relate and potentially help to explain covert attentional cueing.

\section{Experiment 1}

\section{Method}

Participants Twenty young adults, ranging in age from 19 to 26 years $(M=22.4$ years, $S D=3.5$ years, 10 females, two left-handed) were recruited from the local student population of the University of Trento and were tested in the $\mathrm{CIMeC}$ psychophysics laboratories. All participants had normal or corrected-to-normal vision. This research was conducted according to the principles expressed in the Declaration of Helsinki, and informed consent of each participant was obtained. The study was approved by the Ethical Committee of the University of Trento (ethical approval code: 2016-029), and all participants received either a reimbursement of $7 €$ /hour or university credits for their participation. Though our methodological approach to the question is novel, based on previous work that has looked at covert central cueing with similar stimuli (Blair, Capozzi, \& Ristic, 2017; Heimler et al., 2015, Experiment 2; Tipples, 2002), we calculated an estimation of the effect size. Main effects of cue validity and cuetarget SOA reported in Blair et al. (2017) had effect sizes of $\eta_{\mathrm{p}}{ }^{2}=.53$ and $\eta_{\mathrm{p}}{ }^{2}=.63$, respectively. To obtain a desired statistical power of .90 for these main effects corrected for publication bias (Anderson, Kelley, \& Maxwell, 2017), and with an alpha value of .05 , a minimum sample size of 12 individuals was required. Hence, our sample of 20 observers was appropriate for testing these effects.

Stimuli The experiment was run on a 23-inch Asus VG 236 LCD monitor $(100 \mathrm{~Hz}$; resolution 1,920 × 1,080 pixels) that was set up with a viewing distance of $60 \mathrm{~cm}$. Stimulus presentation was controlled by an AMD Radeon Graphics FirePro V4900 graphics card. Luminance values were set using a Minolta CS-100A luminance meter. The background 
was presented in black with a luminance of $0.17 \mathrm{~cd} / \mathrm{m}^{2}$ (RGB: $0,0,0)$. A fixation dot was presented at the center of the screen at the beginning of each trial and served to perform the drift correction. The target, distractor, and arrow stimuli were light gray (RGB: 198, 197, 203) with a luminance of $47 \mathrm{~cd} / \mathrm{m}^{2}$.

Target and distractor consisted of two $90^{\circ}$ rotated squares (width $1^{\circ}$ of visual angle) and were presented at an eccentricity of $11^{\circ}$ from the center of the screen, whereas the face and the arrow were centered $\left(7.2^{\circ} \times 11.5^{\circ}\right.$ and $2.7^{\circ} \times 1.3^{\circ}$ width $\times$ height, respectively). The distractor was always a $90^{\circ}$ rotated whole square, whereas the target could have the bottom or the top part missing; the missing part was a triangle, whose height was one fourth of the total height of the square (see Fig. 1b). All stimuli except the face stimulus were created using OpenSesame (Mathôt, Schreij, \& Theeuwes, 2012), and the experiment itself was run using OpenSesame, together with the PyGaze library (Dalmaijer, Mathôt, \& Van der Stigchel, 2014) and the PsychoPy back end (Peirce, 2007, 2009) to synchronize the PC with the eye-tracker host PC (OpenSesame Version 3.1.3; PyGaze 0.6.0a21). The face was taken from a face database (Oosterhof \& Todorov, 2008). The two directional gaze images (gaze left and right) were created from the straight-ahead gaze (see Fig. 1a) using Photoshop.

Procedure and design Participants were seated on an adjustable chair in front of the monitor in a dimly lit room. Head movements were controlled by means of a chin rest. Participants' gaze was tracked throughout the experiment using an SR Research Ltd., Eyelink 1000 PLUS, consisting of an eye-tracking system connected to a laptop (host PC). It collected monocular gaze position at a sampling rate of $1000 \mathrm{~Hz}$ from the left eye. A five-points-grid calibration routine was completed at the beginning of each experimental condition and at the end of the third block in each experimental condition, for both gaze-cue and arrow-cue conditions. Therefore, a total number of four calibration routines were executed throughout the experiment; additional calibrations were added when necessary (e.g., poor recording due to glasses or contact lens reflection).

Participants were told to fixate the central fixation dot at the beginning of each trial, to try and maintain fixation, and to ignore the cues because they were uninformative. After the drift correction procedure, to make sure that participants' gaze was at the center of the screen, the trial began. Depending on the cue-type condition, which was presented in different blocks, a face with a straight-ahead gaze or a horizontal line was shown for $1,000 \mathrm{~ms}$. This neutral stimulus was followed by the presentation of the directional cue, which consisted of either a face looking to the left or to the right side or an arrow pointing to the left or right side. Crucially, cue direction for both the gaze cue and arrow cue was not predictive of target location. On half of the trials the target appeared on the side indicated by the cue (valid trials), and in the remaining half of

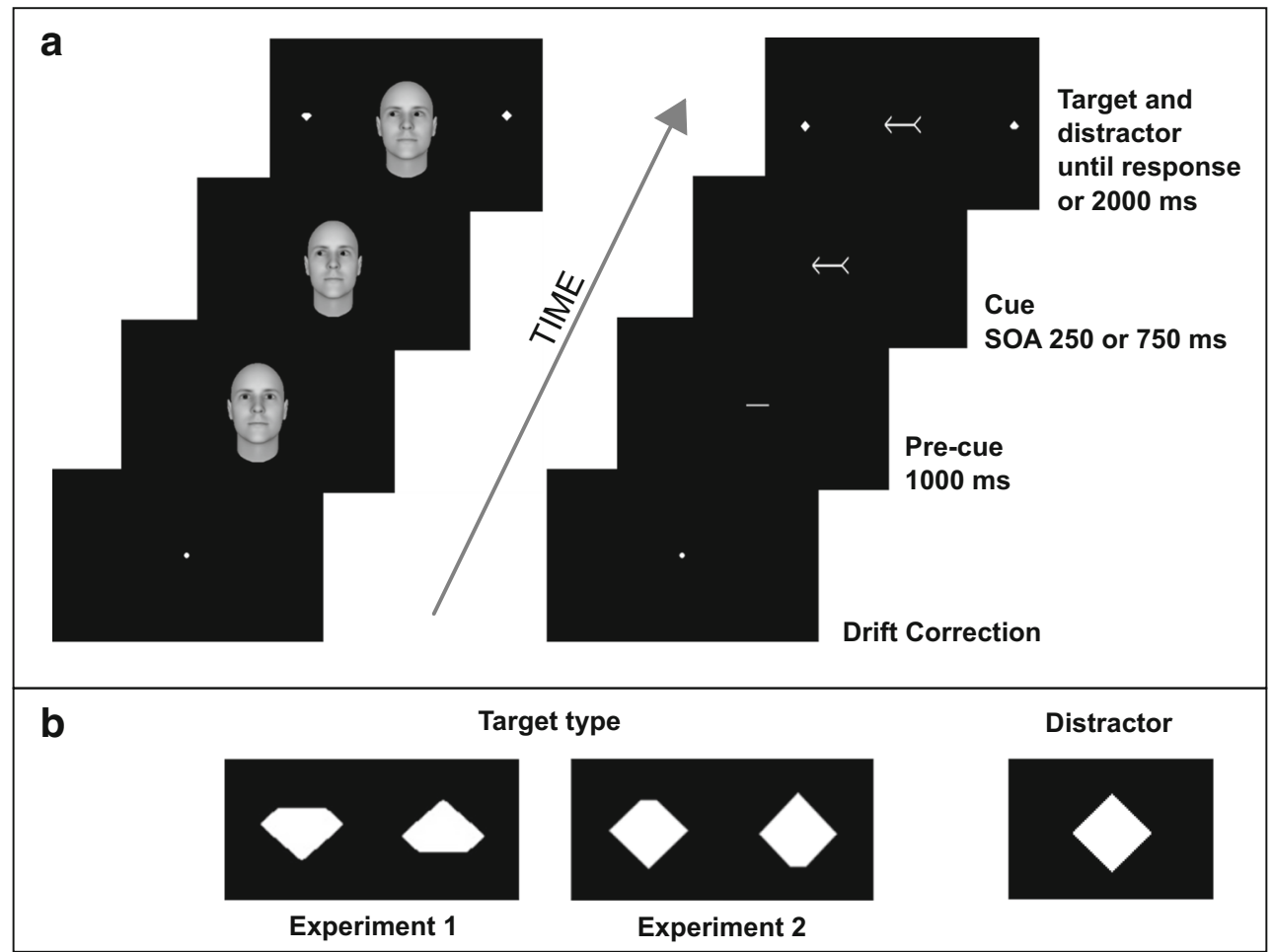

Fig. 1 a Stimuli (not in scale) used in Experiments 1 and 2, as well as main trial sequence. b Target shapes used in the easy (Experiment 1) and difficult (Experiment 2) discrimination task. The distractor shape was always a whole diamond 
trials the target appeared on the opposite side (invalid trials). Two SOAs ( $250 \mathrm{~ms}$ or $750 \mathrm{~ms}$ ) between the cue and target appearance were used in a randomized fashion to detect any changes in the cueing effects as a function of time (Friesen \& Kingstone, 1998; Heimler et al., 2015; Ristic et al., 2002). The task of the participants was to discriminate whether the target was cut on the top or on the bottom part, while keeping fixation at the center of the screen. Participants responded by pressing the $u p$ or down arrow buttons on the computer keyboard with the index and middle fingers of their dominant hand. Participants were instructed to respond as fast and as accurately as possible, indicating whether the target missed the top or the bottom part, independently of the side of the screen in which the target appeared (see Fig. 1a). The experiment comprised 16 practice trials and 160 experimental trials divided in five blocks for each condition (gaze vs. arrow). Hence, participants completed a total number of 32 practice and 320 experimental trials. Cue-type condition was counterbalanced across participants. At the end of each block, participants received feedback on the average RTs and on the percentage of correct responses and were free to take a break. Written instructions and visual sketches of the trial sequence were shown on the screen before starting the practice and were reiterated before starting each experimental condition. As our aim was to investigate whether eye movements would spontaneously occur in this typical covert attention experiment, participants did not receive any feedback when they failed to maintain fixation, and no additional feedback related to eyemovement performance was provided.

\section{Results}

We conducted confirmatory analysis on manual RTs as well as exploratory analyses on specific measures of interest (i.e., saccadic frequency, amplitude, and latency). Descriptive and inferential analyses were conducted using MATLAB (The MathWorks, Version R2016b), STATISTICA (Statsoft, Inc. 2004, Version 7.0), and JASP (JASP Team, 2018, Version 0.8.5.1, Windows 10). For all the dependent measures investigated, the distribution of errors was evaluated to test for the assumption of normality using Shapiro-Wilk normality test as well as for the assumption of homogeneity of variances using Levene's test. Unless otherwise noted, the data confirmed the assumptions.

\section{Patterns of eye movements}

Initial saccades were defined as eye movements with velocities and accelerations exceeding $30 \%$ and $8,000 \% \mathrm{~s}^{2}$. Vertical eye movements, neither directed toward the target nor the distractor, as well as eye movements with no landing coordinates, were removed from the analysis $(2.48 \%$ in gaze-cue condition and $0 \%$ in the arrow-cue condition). In the analyses we included saccades that were executed in trials where a correct manual response to the target was given. To get a general idea of whether eye movements were elicited, and if so, when they occurred relative to the presentation of the cue and following presentation of the target, a frequency distribution of initial eye movements was plotted for the duration of the trial, starting from the moment in which the cue was displayed (see Fig. 2a).

A first visual inspection of this figure reveals the shape of a bimodal distribution for each of the two SOA conditions. It seems that the early peaks of eye movements were triggered by the mere presence of the cue on the screen, from now on referred to as cue-elicited eye movements, while the later peaks of eye movements were executed in response to the presentation of the target and distractor, from now on referred to as target-elicited eye movements. In the short SOA-that is, $250 \mathrm{~ms}$ - the first peak of eye movements was observed after the cue onset within the range of 150 and $350 \mathrm{~ms}$ (median value $=226 \mathrm{~ms}$ ), while a second, higher peak appeared later on, with a median value of $471 \mathrm{~ms}$. In the long SOA — namely, $750 \mathrm{~ms}$ - the minor peak of eye movements after the cue onset had its median value at $265 \mathrm{~ms}$ and the higher peak at $961 \mathrm{~ms}$.

For the cue-elicited saccade distributions, we included all eye movements whose latency ranged from $150 \mathrm{~ms}$ up to $350 \mathrm{~ms}$ after the cue onset (see Fig. 2a) for both SOAs alike. Based on visual inspection of the distributions, we observed that the cue-elicited distribution started at $150 \mathrm{~ms}$ and there was a dip between the two distributions that occurred at 350 $\mathrm{ms}$. For the target-elicited distributions, the interval appeared different for the short SOA compared with the long SOA. In the short SOA, the cue-elicited and target-elicited distributions were very close together and overlapping. The dip that occurred at $350 \mathrm{~ms}$ corresponded with a lower criterion of $100 \mathrm{~ms}$ for the target-elicited saccades (see Fig. 2a). This lower latency boundary was considered to be the one that best separated the two distributions, although it is plausible that a partial overlap between late cue-elicited and fast target-elicited eye movements may be present. In the long SOA condition, the distribution of target-elicited saccades seemed to develop relatively earlier, and ranged from $80 \mathrm{~ms}$ after target onset until RT. Based on these clear bimodal frequency distributions we split the data collected accordingly, and we ran separate statistical analyses for cue-elicited and target-elicited eye movements.

\section{Overall proportion of eye movements}

The total proportions of eye movements were calculated by dividing the actual number of saccades executed by each participant by the number of trials remaining after filtering out incorrect, too fast, and slow responses (see criteria described in the next section). Participants executed a saccade in response to the gaze cue on $19.5 \%(S D=16.2 \%)$ of trials, 


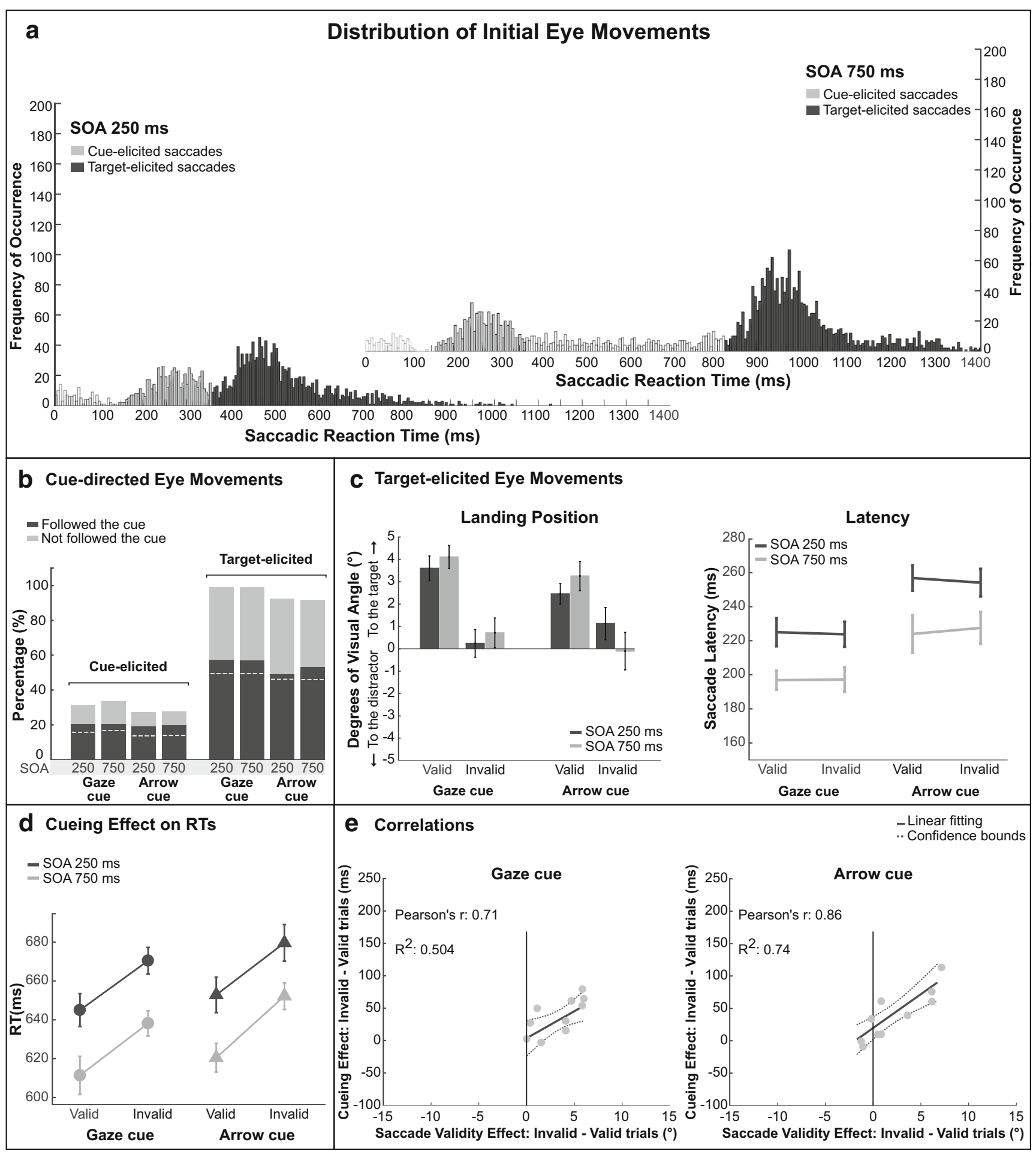

Fig. 2 a Distributions of initial eye movements over time in Experiment 1 , collapsed across cue condition. The zero values on the $x$-axis represent the cue onset, and raw frequencies of occurrence are represented on the $y$ axis. b Mean percentages of cue-directed eye movements in the eyemovement (EM) group $(N=10)$ on short and long SOAs in gaze-cue and arrow-cue conditions. The white dashed line in each bar represents chance level at $50 \%$. c Left: Averaged target-elicited saccade landing positions on valid and invalid trials for gaze-cue and arrow-cue conditions in the EM group. On the $y$-axis, in degrees of visual angle, positive values represent saccades that landed closer to the target and negative numbers indicate saccades that landed closer to the distractor. Right: Mean onset latencies for target-elicited saccades on valid and invalid trials for eyegaze and arrow-distractor conditions in the EM group. $\mathbf{d}$ Mean response times of correct responses to the target on valid and invalid trials for gazecue and arrow-cue conditions in Experiment 1. e Correlations between validity effect on target-elicited saccades against cueing effect on manual RTs for gaze-cue and arrow-cue conditions in the EM group. Descriptive Pearson's $r$ values, coefficients of determination $R^{2}$, as well as a linear fitting with $95 \%$ confidence bound are represented. All error bars denote within-subject standard errors, based on O'Brien and Cousineau (2014) 
whereas they moved the eyes in response to the arrow cue on $17.8 \%(S D=13.8 \%)$ of trials. When we conducted a pairedsamples Wilcoxon test to check for significant effects of cue type, the analysis did not highlight any significant differences between social and nonsocial cues $(\mathrm{W}=125, p=.47)$. On presentation of the target and distractor, the percentage of eye movements elicited increased. With respect to the targetelicited distributions, participants moved their eyes on average in $58.7 \%(S D=42.7 \%)$ of trials in the gaze-cue condition, while they did so in $53.6 \%(S D=43 \%)$ of trials in the arrow-cue condition. We repeated the same Wilcoxon analysis for target-elicited proportions, and no difference between cues emerged $(\mathrm{W}=114, p=.22)$. Note that the large amount of variation in the percentage of eye movements highlights that the overall proportion of eye movements varied dramatically across participants. The results showed that there were some participants who made eye movements in nearly all trials, whereas some participants maintained fixation at the center of the display in the majority of trials.

\section{Behavioral cue effect on RT}

The overall accuracy rate was 97\%. Incorrect responses, RTs three standard deviations above the participant's average RT, and timed-out trials were excluded from the analysis. The total percentage of trials discarded from the analysis was $4.25 \%$ for the gaze-cue condition and $3.78 \%$ for the arrow-cue condition. Mean RTs were calculated, and the distribution of error was evaluated to meet the assumptions for a repeated-measures ANOVA. A $2 \times 2 \times 2$ repeated-measures ANOVA, with cue type (gaze vs. arrow), SOA (250 vs. $750 \mathrm{~ms}$ ), and validity (valid vs. invalid), was run. Main effects of SOA, $F(1,19)=$ $29.54, p<.0001, \eta_{\mathrm{p}}{ }^{2}=.61$, and validity, $F(1,19)=29.68, p<$ $.0001, \eta_{\mathrm{p}}{ }^{2}=.61$, were found. Valid trials $(M=632 \mathrm{~ms}, S E=$ 19.8) were responded to faster than were invalid trials $(M=$ $660, S E=22.3)$, and trials in the longer SOA $(M=630 \mathrm{~ms}, S E$ $=20.3)$ were responded to faster than in the shorter SOA $(M=$ $662, S E=22$; see Fig. 2d). No other significant effect or interaction was found (all $F s<.81, p s>.37$ ).

Because we were interested in the potential modulation of eye movements on manual RT, the behavioral RT data were also analyzed in dependency of whether or not participants made eye movements in response to the target presentation. Each participant was categorized based on the presence of eye movements after target onset; if, on average, 15 data points per cell were available in one of the categories (i.e., eye movements vs. no eye movements), then that participant's data were added to the appropriate group. This led to a surprisingly even split, with 10 participants in the so-called eye-movement group (EM group, on average eye movements in $98.7 \%$ of trials for the gaze-cue and $91.9 \%$ for the arrow-cue condition) and 10 participants in the no-eye-movement group (no-EM group, $18.6 \%$ of trials with eye movements for the gaze-cue condition and $15.3 \%$ for the arrow-cue condition). A mixed ANOVA, with group as a between-subjects factor and cue type, SOA, and validity as within-subjects factors was run on RTs. The group comparison revealed significant main effects of SOA, $F(1,18)=33.12, p<.001, \eta_{\mathrm{p}}{ }^{2}=.65$, and validity across both groups, $F(1,18)=30.95, p<.001, \eta_{\mathrm{p}}{ }^{2}$ $=.63$, though the validity effect was larger in the EM group $(M$ $=38.15 \mathrm{~ms})$ compared with the no-EM group $(M=15.4 \mathrm{~ms})$, as indicated by a significant two-way interaction between group and validity, $F(1,18)=5.59, p=.03, \eta_{\mathrm{p}}{ }^{2}=.24$. In addition, participants in the EM-group responded slower than those who did not (main effect of group), $F(1,18)=7.13, p=$ $.02, \eta_{\mathrm{p}}{ }^{2}=.28$.

\section{Cue-elicited eye movements}

General direction This analysis concerns the proportion of initial eye movements made in response to the nonpredictive cues, prior to the target onset. Since the data from the no-EM group were too noisy, given that there were very few sample points per cell, analyses were conducted on the EM group only. For the EM group, we calculated proportions of eye movements made in accordance to the direction indicated by the gaze and arrow cue. The results showed that in the short SOA, participants overtly followed the direction of the gaze cue in $66 \%$ of all trials and overtly followed the arrow cue in $68 \%$ of all trials. A similar result was found in the longer SOA, where observers followed the gaze cue in $62 \%$ and the arrow cue in $72 \%$ of the trials (see Fig. 2 b for more details on the EM group). We conducted a repeated-measures ANOVA on the proportions of cue-followed eye movements to check whether cue type affected the number of eye movements elicited in response to the cue, but no significant effect was found (all $F \mathrm{~s}<0.8, p \mathrm{~s}>.4)$.

Saccade latency The data set on latency included all initial saccades in the EM group, whose latency was above the lower boundary, set at $150 \mathrm{~ms}$. A $2 \times 2$ repeated-measures ANOVA on the average saccadic reaction time was conducted, with cue type (gaze vs. arrow) and SOA (250 vs. $750 \mathrm{~ms}$ ) as withinsubjects independent variables (only one condition failed to satisfy the assumption of normality, with $p=.004)$. A main effect of cue type, $F(1,9)=7.67, p=.02, \eta_{\mathrm{p}}{ }^{2}=.46$, was found. Gaze-cue condition triggered faster eye movements $(M=256$ $\mathrm{ms}, S E=5.05)$ than the arrow cue $(M=270 \mathrm{~ms}, S E=4.4)$. No other main effects or interaction reached significance (all $F_{S}<$ $.60, p \mathrm{~s}>.45)$.

\section{Target-elicited eye movements}

Saccade landing position Saccade landing position was obtained measuring the distance in degrees of visual angle between the $x$ and $y$ coordinates of the target center and the 
ending coordinates of each saccade, such that $0^{\circ}$ of visual angle represented an eye movement that landed on the target, and $22^{\circ}$ represented an eye movement that landed on the distractor (note that in Fig. 2c the measure is rereferenced to a scale of signed values for clarity purposes; positive values represent saccades that landed closer to the target, negative values represent values closer to the distractor). Outliers were removed by means of a three standard deviations of the mean criterion. A $2 \times 2 \times 2$ repeated-measures ANOVA on the average distance from the target in degrees of visual angle was run. Cue type (gaze vs. arrow), SOA (250 ms vs. 750 $\mathrm{ms}$ ), and validity (valid vs. invalid) were the within-subjects independent variables. A main effect of validity, $F(1,9)=$ $15.2, p=.004, \eta_{\mathrm{p}}{ }^{2}=.63$, was found. Valid trials $\left(M=7.75^{\circ}\right.$, $S E=.53)$ resulted in larger saccades towards the validly cued target position compared with invalid trials $\left(M=10.6^{\circ}, S E=\right.$ .88). No other main effect or interaction reached significance (all $F s<3.28, p s>.1$ ).

Saccade latency Saccade latency was defined as the time between the target onset and the initiation of a saccade. Saccade latencies smaller than and $100 \mathrm{~ms}$ for the short SOA and $80 \mathrm{~ms}$ for the long SOA, together with latencies slower than three standard deviations from the mean of each participant, were not taken into consideration for further analysis. A $2 \times 2$ $\times 2$ repeated-measures ANOVA on the average saccadic reaction times was run, with cue type (gaze vs. arrow), SOA (250 ms vs. $750 \mathrm{~ms}$ ), and validity (valid vs. invalid) as within-subjects independent variables. A main effect of cue type, $F(1,9)=5.86, p=.04, \eta_{\mathrm{p}}{ }^{2}=.39$, and SOA, $F(1,9)=$ $22.12, p=.001, \eta_{\mathrm{p}}{ }^{2}=.71$, was found (see Fig. $2 \mathrm{c}$ ). On average, gaze cue triggered faster eye movements $(M=211 \mathrm{~ms}, S E$ $=11.2)$ compared with the arrow cue $(M=241 \mathrm{~ms}, S E=19.6)$. In trials where the SOA was shorter, average saccade RT was longer $(M=240 \mathrm{~ms}, S E=14.8)$ than in trials with longer SOA $(M=211 \mathrm{~ms}, S E=15.2)$. No other main effects or interaction reached significance (all $F s<1.38, p s>.27$ ).

\section{Correlations}

To directly explore the relationship between overt performance and behavioral effect on manual RTs, we plotted the relationship between the validity effect on manual RTs against the validity effect on landing positions of target-elicited saccades for the EM group. To this end, we considered only trials where both RT and eye-movement data were available. The validity effect on RT was calculated by subtracting the average RT on valid trials from the average RT in invalid trials. Following the same procedure, we computed the validity effect on target-elicited distance to target by subtracting the values in valid trials from the corresponding values in invalid trials. Strong positive linear correlations for both gaze-cue, Pearson's $r(10)=.71,95 \%$ CI $[.14, .92], R^{2}=.50$, and arrow-cue condition, Pearson's $r(10)=.86,95 \%$ CI $[.50$, .97], $R^{2}=.75$, were found (see Fig. 2e). In other words, for both cues, the relationship indicates that the greater the difference in saccadic landing position in response to invalid and valid cues, the greater the difference in RT between invalid and valid cues.

\section{Discussion}

In Experiment 1, we investigated whether overt orienting can help explain behavioral manual responses towards social and nonsocial cues. Participants performed a discrimination task and were presented with nonpredictive central gaze and arrow cues at two different cue-target SOAs. There were three main findings. First, our data replicated previous and welldocumented cueing effects on response times (Driver et al., 1999; Friesen \& Kingstone, 1998; Galfano et al., 2012; Heimler et al., 2015; Langton \& Bruce, 1999; Ristic et al., 2002). Although participants were aware of the fact that both cues were nonpredictive, they responded faster to valid cues compared with invalid cues. In addition, when the interval between the cue and the target was longer, behavioral responses were faster. This latter result is a well-known phenomenon called cue-target foreperiod effect and reflects a general preparatory process (Bertelson, 1967). We found no significant difference in the magnitude of the cueing effect of the gaze versus the arrow cue, suggesting that the two cues were similar in the ability to trigger automatic orienting (Stevens, West, Al-Aidroos, Weger, \& Pratt, 2008).

Second, the proportion of eye movements made in Experiment 1 highlighted that for some observers, oculomotor responses were not necessary to perform the task accurately. Nevertheless, even if participants were asked to maintain fixation throughout the experiment, our results show that saccadic eye movements were often made during the task. In particular, a group of individuals triggered eye-movement responses before their manual response. While in the majority of cases cue-elicited eye movements were found to follow the direction indicated by the uninformative cues, target-elicited saccades generally landed closer to valid targets only. Invalid trials did not elicit a reliable overt response, neither to the distractor nor to the target. Although cue type did not appear to affect behavioral responses or landing positions, an effect of cue type emerged with respect to saccade latency, both for cue-elicited and target-elicited movements. The results showed that saccades initiated in response to gaze cues were generally faster than saccades elicited in response to arrow cues. In this sense, the saccade latency data supported the hypothesis that overt orienting in response to a gaze cue is more reflexive compared with responses to an arrow cue. Additionally, target-elicited saccade latencies were also modulated by SOA, as saccadic reaction times (SRTs) were slower in the short SOA. The pattern was consistent with the foreperiod effect found in the 
behavioral data. However, critically, saccade latency was not modulated by validity, and saccades were equally fast regardless of whether the cue was valid or invalid.

Third, linear correlations between the validity effect on target-elicited saccades and the manual validity effect on RT disclosed a strong association between overt and covert performance for both cue conditions. The amplitude of saccades made in response to invalid and valid trials was strongly associated to the magnitude of the validity effect on RTs. However, we are aware of the fact that the main limitation of this finding in Experiment 1 is that it is based on only half of the experimental sample, as only half the sample provided sufficient eye-movement data.

To sum up, Experiment 1 allowed us to make a first inspection of the relative contribution of overt selection during a simple discrimination task. Oculomotor responses were associated with overall slower manual RTs and, when present, they landed closer to the valid target location. In addition, manual responses and eye-movement landing position patterns did not differ in dependency of the cue, suggesting that the overt mechanism of selection operates similarly irrespective of the nature of the central cue presented. Though saccade latencies were affected by the type of cue presented, this difference between social and nonsocial cues was not carried over to the manual RTs. This may be the case because eye movements were not instrumental in correctly identifying the target. In fact, the strong relationship between eye movements and the cueing effect on RT was found in 10 out of 20 participants, suggesting that individual differences played an important role in the final oculomotor behavior. While the sample size of 20 participants proved sufficient in terms of power for manual measures, in terms of saccadic dependent measures, it was ambivalent, that is, it was appropriate for saccade amplitude, but not fully convincing for saccade latency. Hence, this further motivated Experiment 2, where we increased the need for eye movements yielding more data for these dependent measures.

\section{Experiment 2}

To further investigate the association between oculomotor responses and orienting of spatial attention to social and nonsocial cues, we designed a second experiment. The aim of the second experiment was to make eye movements instrumental in the task, while keeping experimental instructions identical. We manipulated the difficulty of the discrimination task by reducing the size of the missing part of the target diamond. Because identification of the missing part of the target would benefit from closer foveal scrutiny, we hypothesized that this difficulty manipulation would lead to an overall increase in the rate of eye movements. We also speculated that saccades could be biased more strongly by the central cues. We further hypothesized that the increased difficulty of the task would slow down overall RT and possibly decrease accuracy, but that the overall manual cueing effect should be similar compared with Experiment 1. Again, if saccade landing position and manual RT are associated, we should be able to replicate the strong correlation found in Experiment 1.

\section{Method}

Participants Twenty new young adults ranging in age from 20 to 36 years $(M=25.6$ years, $S D=4.4$ years, 10 females, all right-handed) were recruited from the local student population of the University of Trento and were tested in the CIMeC psychophysics laboratories. All participants had normal or corrected-to-normal vision. This research was conducted according to the principles expressed in the Declaration of Helsinki, and informed consent was obtained from each participant. All participants received either a reimbursement of $7 €$ /hour for their participation or University credits. To ensure that our experiment had enough statistical power to test the presence of a relationship between saccadic and manual validity effects found in Experiment 1, effect sizes of validity on manual RT $\left(\eta_{\mathrm{p}}{ }^{2}=.61\right)$ and on saccadic amplitude $\left(\eta_{\mathrm{p}}{ }^{2}=.63\right)$ from Experiment 1 were used to estimate the new effect size. With a minimum sample size of 11 participants, a desired statistical power of .90 for these effects on validity could be reached, correcting for publication bias (Anderson et al., 2017). As in Experiment 1, our sample of 20 observers was appropriate for testing these effects.

Stimuli, procedure, and design The monitor was replaced between experiments. Stimuli in the second experiment were presented on a 23.6-inch ViewPixx EEG monitor $(100 \mathrm{~Hz}$; resolution $1,920 \times 1,080)$ that was set up with a viewing distance of $60 \mathrm{~cm}$. Stimulus presentation was controlled by a NVIDIA Quadro K620 graphics card. The remainder of the design, materials, and procedure of Experiment 2 were identical to those in Experiment 1, except for the target stimulus, whose color was slightly changed to maintain the same luminance as in Experiment 1 with the previous screen (RGB: 190, 196, 208). The missing part of the target was a triangle, whose height was one tenth of the target total height (see Fig. 1b).

We modified the target shape to be sure that people could not discriminate it without moving the eyes. However, since we did not want to bias observers into a different strategy, for example, of making more eye movements from the start compared with Experiment 1 or of avoiding eye movements before the actual target onset, we provided participants with the same instructions as in Experiment 1. Therefore, participants were asked to maintain fixation and to be as accurate as possible on the discrimination task. Again, similar to Experiment 1 , no feedback was provided on eye-movement behavior and fixation was by no means reinforced. 


\section{Results}

For all the dependent measures we investigated, the distribution of error was evaluated to test for the assumption of normality using Shapiro-Wilk normality test as well as for the assumption of homogeneity of variances using Levene's test. Unless otherwise noted, the data confirmed the assumptions.

\section{Patterns of eye movements}

Visual inspection of the total frequency of eye movements over time reconfirmed the existence of two bimodal distributions. In the short SOA, we observed a peak of eye movements that followed the cue onset (median value $=219 \mathrm{~ms}$ ) and a second, higher peak after the target appeared on the screen (median value $=412 \mathrm{~ms}$ ). In the long SOA, the two peaks respectively emerged after the cue onset (median value $=$ $268 \mathrm{~ms}$ ) and later on, in response to the target and distractor onset (median value $=906 \mathrm{~ms}$ ). Consequently, we could again discriminate cue-elicited eye movements from target-elicited eye movements (see Fig. 3a). Initial saccades were defined using the same criteria explained in the Results section of Experiment 1 and we selected cue-elicited saccades and target-elicited saccades within the same intervals reported in Experiment 1. We ran the same analysis on the proportion of eye movements, landing position, and latency, discarding a further $0.19 \%$ of trials in the gaze-cue condition and $1.3 \%$ in the arrow-cue condition.

\section{Overall proportion of eye movements}

The total proportions of eye movements were calculated by adopting the criteria reported in Experiment 1. Cue-elicited eye movements were triggered on $33.8 \%(S D=15.6 \%)$ of trials in the gaze-cue condition and on $41.5 \%(S D=19 \%)$ in the arrow-cue condition (see Fig. 3b), and the proportions did not vary as a function of cue type (paired-samples Wilcoxon test, $\mathrm{W}=54, p=.06$ ). In addition, saccades in response to the target and distractor in the gaze-cue condition occurred on average in $99 \%(S D=1.2 \%)$ of trials and in $99.5 \%$ ( $S D=$ $0.8 \%$ ) of trials in the arrow-cue condition; there were no significant differences between cues (paired-samples Wilcoxon test, $\mathrm{W}=27.5, p=.22$ ). Note that the task manipulation almost doubled the overall percentage of eye movements and considerably reduced the variability compared with Experiment 1, showing that the manipulation worked as intended.

\section{Behavioral cue effect on RT}

The overall accuracy rate was again $97 \%$, and therefore no further analysis on accuracy was carried out. Mean RTs were calculated and outliers were removed using a three standard deviation criterion. The proportion of trials discarded from the analysis was $2.90 \%$ for the gaze-cue condition and $2.96 \%$ for the arrow-cue condition. A $2 \times 2 \times 2$ repeated-measures ANOVA with cue type (gaze vs. arrow), SOA (250 vs. 750 $\mathrm{ms}$ ), and validity (valid vs. invalid) was run.

Main effects of cue type, $F(1,19)=29.91, p<.0001, \eta_{\mathrm{p}}{ }^{2}=$ .61 ; SOA, $F(1,19)=33.32, p<.0001, \eta_{\mathrm{p}}{ }^{2}=.64$; and validity, $F(1,19)=29.61, p<.0001, \eta_{\mathrm{p}}{ }^{2}=.61$, were found. Gaze cues $(M=812 \mathrm{~ms}, S E=15.8)$ were responded to faster than arrow cues $(M=868 \mathrm{~ms}, S E=16.5)$, longer SOA $(M=818 \mathrm{~ms}, S E=$ 16.4) were faster than shorter SOA $(M=862 \mathrm{~ms}, S E=16.4)$, and valid trials $(M=807 \mathrm{~ms}, S E=16.3)$ resulted in faster RTs than invalid trials $(M=872 \mathrm{~ms}, S E=16.7$; see Fig. $3 \mathrm{~d})$. No other significant interactions were found (all $F_{\mathrm{S}}<1.21, p \mathrm{~s}>$ $.28)$.

In addition, we directly compared behavioral performance of Experiment 1 and Experiment 2 to see whether the task modulated the validity effect on RTs, since Experiment 2 required participants to move their eyes to identify the target shape. A mixed ANOVA with experiment as a betweensubjects factor and cue type, SOA, and validity as withinsubjects factors was performed on RTs. Main effects of experiment, $F(1,38)=55.48, p<.001, \eta_{\mathrm{p}}{ }^{2}=.59$; cue type, $F(1,38)$ $=18.96, p<.001, \eta_{\mathrm{p}}{ }^{2}=.33$; SOA, $F(1,38)=62.13, p<.001$, $\eta_{\mathrm{p}}{ }^{2}=.62$; and validity, $F(1,38)=50.83, p<.001, \eta_{\mathrm{p}}{ }^{2}=.57$, were found. The increased difficulty of the task had a significant impact on RTs, which were overall slower $(M=840 \mathrm{~ms}$, $S D=18.4)$. Furthermore, a significant two-way interaction between experiment and cue type, $F(1,38)=9.13, p=.004$, $\eta_{\mathrm{p}}{ }^{2}=.19$, revealed that the gaze cue was more beneficial to RTs compared with the arrow cue when the task was more difficult. In addition, the validity effect on RTs was larger in Experiment 2, as pointed out by the significant two-way interaction between experiment and validity, $F(1,38)=8.41, p=$ $.006, \eta_{\mathrm{p}}^{2}=.18$.

This result suggests that task difficulty had a distinct effect on the overall RT of the arrow cue but not the gaze cue in Experiment 2 compared with Experiment 1 . However, rather than being driven by task difficulty, this may be a result of the presence of eye movements, which were more abundant in Experiment 2 than in Experiment 1. The manual results in Experiment 1 contained all participants, including those who made very little eye movements. To test the idea that the eye movements distinctively speed manual RTs to gaze cues but not arrow cues, we compared the manual responses of the EM group of Experiment 1 with all of the participants in Experiment 2. A between-experiment ANOVA on the aforementioned samples, including cue type (gaze vs. arrow) as a within-subjects factor and experiment (1 vs. 2) as a betweensubjects factor was run. The analysis revealed a main effect of cue type, $F(1,28)=25.4, p<.001, \eta_{\mathrm{p}}{ }^{2}=.47$; a main effect of experiment, $F(1,28)=25.41, p<.001, \eta_{\mathrm{p}}{ }^{2}=.47$; but no interaction between cue type and experiment, $F(1,28)=2.5$, $p=.122$, suggesting that when we matched for eye 

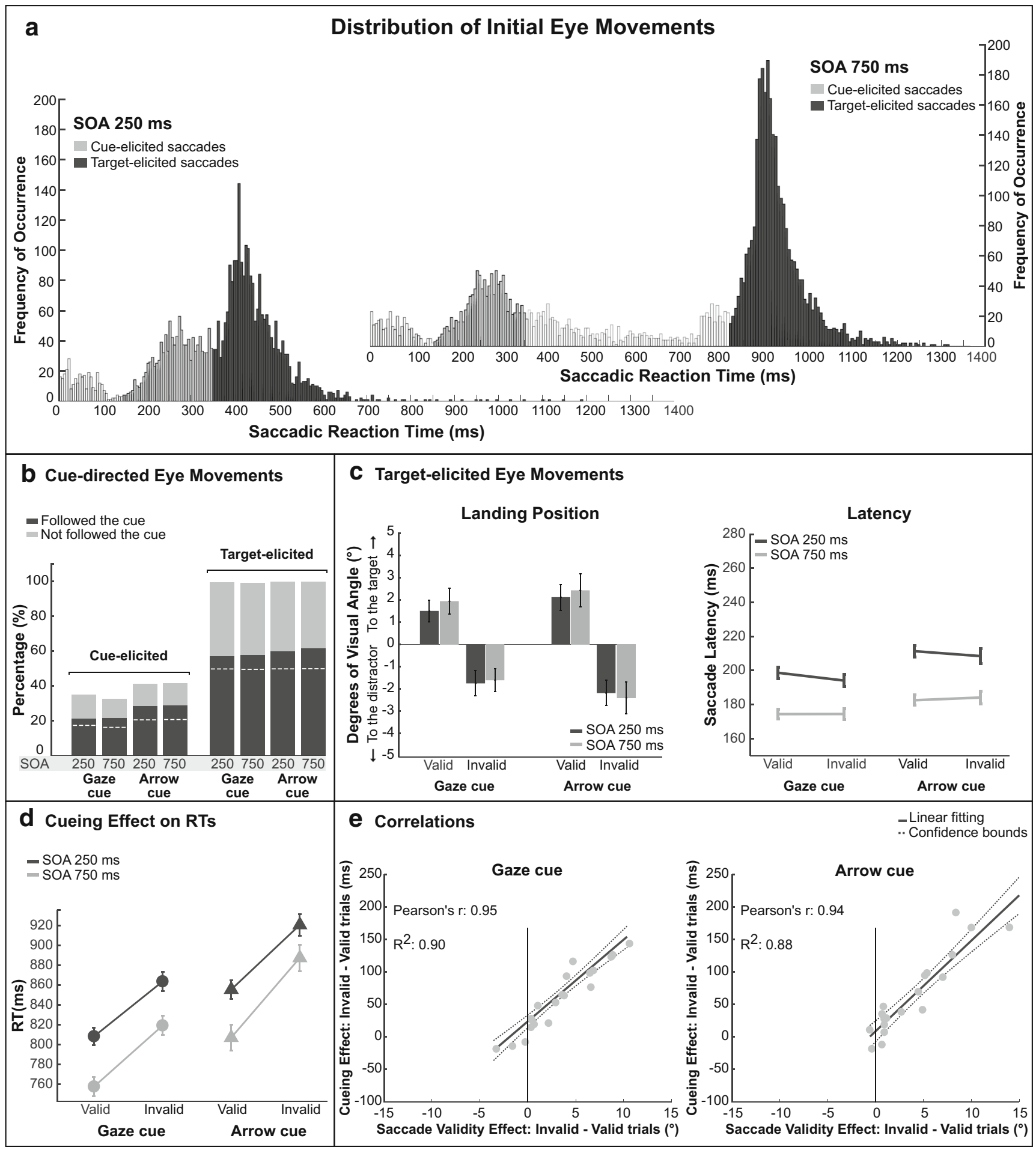

Fig. 3 a Distributions of initial eye movements over time in Experiment 2, collapsed across cue condition. b Mean percentages of cue-directed eye movements on short and long SOAs in gaze-cue and arrow-cue conditions. The white dashed line in each bar represents chance level at $50 \%$. Note that this time all participants were included in the data set. $\mathbf{c}$ Left: Averaged target-elicited saccade landing positions on valid and invalid trials for gaze-cue and arrow-cue conditions in Experiment 2. On the $y$ axis, in degrees of visual angle, positive values represent saccades that landed closer to the target and negative numbers indicate saccades that landed closer to the distractor. Right: Mean onset latencies for target- elicited saccades on valid and invalid trials for eye-gaze and arrow distractor conditions in Experiment 2. d Mean response times of correct responses to the target on valid and invalid trials for gaze-cue and arrowcue conditions in Experiment 2. e Correlations between validity effect on target-elicited saccades against cueing effect on manual RTs for gaze-cue and arrow-cue conditions in Experiment 2. Descriptive Pearson's $r$ values, coefficients of determination $R^{2}$, as well as a linear fitting and $95 \%$ confidence bounds are also depicted. All error bars represent withinsubject standard errors, based on O'Brien and Cousineau (2014) 
movements, both experiments highlighted a comparable gazecue advantage on overall RTs. When the EM group was considered in isolation, a marginally significant cue-dependent difference in overall RT was found (repeated-measures ANOVA), $F(1,9)=4.94, p=.053$, that was not present in the no-EM group. In other words, the social cue tended to trigger faster manual responses in the EM group also in Experiment 1.

\section{Cue-elicited eye movements}

General direction We calculated the proportion of eye movements directed towards the location indicated by the gaze and arrow cues, for both SOAs (see Fig. 3b). In the short SOA, participants followed the cue on $62 \%$ (gaze-cue condition) and $69 \%$ (arrow-cue condition) of trials, respectively. Similarly, in the longer SOA the proportion in percentage were $72 \%$ (gaze cue) and 68\% (arrow cue). The repeatedmeasures ANOVA on the proportions of cue-followed eye movements did not highlight any significant effects of cue type or SOA (all $F \mathrm{~s}<2.03, p s>.17$ ) on the general direction of cue-elicited saccades.

Saccade latency As we did in Experiment 1, in the analysis we considered all saccadic RTs longer than $150 \mathrm{~ms}$. A $2 \times 2$ repeated-measures ANOVA on the saccadic RT was performed, with cue type (gaze vs. arrow) and SOA (250 vs. $750 \mathrm{~ms}$ ) as within-subjects independent variables. A main effect of cue type, $F(1,19)=11.84, p=.003, \eta_{\mathrm{p}}{ }^{2}=.38$, was found. The gaze-cue condition triggered faster eye movements $(M=262 \mathrm{~ms}, S E=3.32)$ than did the arrow-cue condition $(M=275 \mathrm{~ms}, S E=3.51)$. No other main effects or interactions reached significance (all $F \mathrm{~s}<.29, p \mathrm{~s}>.59$ ).

\section{Target-elicited eye movements}

General direction We calculated the proportion of eye movements directed towards the location indicated by the gaze and arrow cue, for both SOAs (see Fig. 3b). In the short SOA, participants followed the cue on $62 \%$ (gaze-cue condition) and $69 \%$ (arrow-cue condition) of trials, respectively. Similarly, in the longer SOA, the proportions in percentage were $72 \%$ (gaze cue) and $68 \%$ (arrow cue).

Saccade landing position We followed the same procedure of Experiment 1 to calculate saccade landing position. A $2 \times 2 \times$ 2 repeated-measures ANOVA was conducted on the average distance from the target in degrees of visual angle; cue type (gaze vs. arrow), SOA (250 ms vs. $750 \mathrm{~ms}$ ), and validity (valid vs. invalid) constituted the within-subjects independent variables (see Fig. 3c). A main effect of validity, $F(1,19)=22.97$, $p<.001, \eta_{\mathrm{p}}{ }^{2}=.55$, was found. Saccades in valid trials $(M=$ $9.39^{\circ}, S E=.45$ ) landed closer to the target, whereas saccades in invalid trials landed closer to the distractor $\left(M=13.36^{\circ}, S E\right.$ $=.46)$. No other main effect or interaction reached significance (all $F \mathrm{~s}<1.45, p \mathrm{~s}>.24$ ).

In addition, we compared Experiment 1 and Experiment 2 with respect to the landing position of eye movements to see whether the difficulty of the task had an impact on saccade amplitude. A mixed ANOVA with Experiment (1 vs. 2) as between-subjects factors and cue type (gaze vs. arrow) and validity (valid vs. invalid) as within-subjects factors was performed. A main effect of validity, $F(1,28)=28.09, p<.001$, $\eta_{\mathrm{p}}{ }^{2}=.50$, was present. Saccades landed closer to the target in valid trials $\left(M=8.57^{\circ}, S E=.37\right)$ compared with invalid trials $\left(M=11.98^{\circ}, S E=.45\right)$. Furthermore, a main effect of experiment, $F(1,28)=18.84, p<.001, \eta_{\mathrm{p}}{ }^{2}=.40$, was found. In Experiment 1, we confirmed that landing positions of initial eye movements were biased to the valid target position only $\left(M=9.17^{\circ}, S E=.41\right)$, while in Experiment 2 initial eye movements followed the position indicated by the nonpredictive cue $\left(M=11.38^{\circ}, S E=.29\right)$. No other main effect or interaction reached significance (all $F \mathrm{~s}<2.05, p \mathrm{~s}>.16$ ).

Saccade latency In order to satisfy the assumption of normality, we performed a logarithmic transformation of the latency data set (Shapiro-Wilk normality test, all $p$ s >.161). A $2 \times 2 \times$ 2 repeated-measures ANOVA was run, with cue type (gaze vs. arrow), SOA (250 ms vs. $750 \mathrm{~ms}$ ), and validity (valid vs. invalid) as within-subjects factors. This analysis revealed a main effect of cue type, $F(1,19)=6.64, p=.018, \eta_{\mathrm{p}}{ }^{2}=.26$, and SOA, $F(1,19)=68.72, p<.0001, \eta_{\mathrm{p}}{ }^{2}=.78$ (see Fig. $3 \mathrm{c}$ ). Faster saccades were triggered by the gaze cue $(M=183 \mathrm{~ms}$, $S E=1.032)$ compared with the arrow cue $(M=193 \mathrm{~ms}, S E=$ 1.036). In trials where the SOA was shorter, average saccade RT was slower $(M=201 \mathrm{~ms}, S E=1.03)$ than in trials with longer SOA $(M=176 \mathrm{~ms}, S E=1.034)$. No other main effects or interactions were significant (all $F_{\mathrm{s}}<2.8, p \mathrm{~s}>.11$ ).

\section{Correlations}

To investigate the direct relationship between eye movements and cueing effect on RT, we plotted the relationship between the validity effect on manual RTs against the validity effect on landing positions of target-elicited saccades. We followed the criteria of Experiment 1, but this time all participants provided sufficient data and were all included in the analysis. Again, we calculated the validity effect on RTs and target-elicited distance to the target by subtracting the values in valid trials from the corresponding values in invalid trials. Strong positive linear correlations for both gaze, Pearson's $r(20)=.95$, $95 \%$ CI $[.87, .98], R^{2}=.90$, and arrow-cue condition, Pearson's $r(20)=.94,95 \%$ CI $[.85, .98], R^{2}=.88$, were found, confirming the findings of Experiment 1, but this time in a larger data set (see Fig. 3e). 


\section{Discussion}

In Experiment 2, the discrimination task was made more difficult in order to make eye movements instrumental in the task. The results showed that in comparison to Experiment 1, the proportions of eye movement increased substantially. Taken together, these findings indicate that the task manipulation effectively raised the need for overt shifts. In addition, relative to Experiment 1, overall RTs slowed down, indicating that participants needed more time to resolve the identity of the target. Interestingly, the behavioral performance highlighted a significant difference between cue conditions, where observers were faster to respond when the nonpredictive cue was the eye gaze. However, this new cue-dependent difference was unlikely due to task difficulty and Experiment 2 being more difficult. When we checked the results against those of Experiment 1 by looking at only the observers who made eye movements $(N=10)$, we found a very similar difference in Experiment 1, in that gaze was responded to faster than was the arrow. Thus, regardless of difficulty, in the presence of eye movements, manual responses to gaze stimuli were faster than to arrow stimuli, which is in line with the general results on saccade latency.

Looking at the pattern of eye movements, the analysis of the cue-elicited eye movements confirmed that participants tended to follow the cue. Saccade landing positions in the target-elicited distribution were affected by cue validity. Neither the cue-elicited nor the target-elicited saccades were affected by the type of cue, whether it was a gaze or arrow cue. Unlike the results in Experiment 1, invalid cues elicited an overt response away from the target in the general direction of the distractor in Experiment 2. While the analysis of initial saccade landing positions did not reveal reliable differences between social and nonsocial cues, the analyses on saccade latency did. Prior to target onset, cue-elicited saccades were faster when triggered by the social cue. Similarly, target-elicited saccadic RT varied in dependency of the cue presented, with faster saccadic RTs when the cue was the gaze. Hence, evidence from Experiment 2 is in favor of the notion that social cues may elicit more reflexive shifts in overt attention than do nonsocial cues. In addition, this differentiation seemed to extend up to the final response, in the sense that gaze-cue associated overall RTs were speeded up. However, critically, this difference in saccade latency between cues did not carry over to differences in validity effects on RT, or to differences in landing positions across cues. Finally, correlations between the cueing effect on RT and validity effect on eye movements confirmed the trend shown in Experiment 1, adding converging evidence for a strong association between the initial landing position of the oculomotor response and the final manual response.

\section{General discussion}

In this study we investigated the impact of spontaneous oculomotor behavior on attentional performance. Our approach extends previous research, in the sense that it enabled us to examine oculomotor performance and attentional orienting at different moments in time, and it demonstrates that spontaneous eye movements are present even before the occurrence of a specific event (i.e., the target appearance), and even when the task does not require overt responses.

First, our data reveal the cue had an immediate impact on overt performance that was already present in the cueelicited saccadic responses. In previous work on covert attention, participants' gaze was not tracked, so the impact of the uninformative cues on cue-elicited eye movements could not be measured with RT or inferred from RT patterns. Previous studies on overt attention that revealed potential differences between these cues have focused on target-elicited saccade latencies, not considering the dynamic deployment of attention over time and its consequences on different oculomotor parameters.

Second, we found that cue type has a distinct impact on oculomotor components. While saccade latencies were affected by the type of cue, both before and after the target onset, saccade landing positions were not.

Starting from the 1990s, findings have pointed out that centrally presented nonpredictive cues can trigger reflexive orienting of attention (e.g., Driver et al., 1999; Friesen \& Kingstone, 1998; Galfano et al., 2012; Heimler et al., 2015; Hommel et al., 2001; Langton \& Bruce, 1999; Ristic et al., 2002; Ristic \& Kingstone, 2005; Tipples, 2002; however, see Gibson \& Bryant, 2005; Vecera \& Rizzo, 2004, 2006). In our study, the observed cueing effects on RTs are in line with a wide range of studies that demonstrate how both uninformative gaze as well as arrow cues result in orienting of covert attention, showing that valid trials are responded to faster than invalid trials. Despite the stark perceptual differences between the gaze and arrow cues in terms of complexity, results of Experiment 1 and 2 show that the influence of both nonpredictive cues on spatial attention, in terms of validity effects, was comparable. However, manual RTs represent the final result of orienting of attention and selection. Dynamic visual processing is difficult to access using RTs only. In the present work, we recorded eye movements during the cueing task to examine their temporal evolution and investigated how they contribute or influence the final manual response. Previous work on overt attention did not collect manual RTs and was based on specific instructions, which anchored saccades execution at a certain point in time (Kuhn \& Benson, 2007; Kuhn, Benson, et al., 2009a; Kuhn \& Kingstone, 2009). Differently from previous research on overt attention, the present work focused on the impact that spontaneous oculomotor behavior may have on attentional performance. 
Consequently, we did not specifically and explicitly instruct participants to start an eye movement at a certain point in time or to a specific target. Rather than giving such instructions, we asked participants to try and maintain fixation during the task, similar to previous studies on covert orienting of attention. In Experiment 1, we observed variability in oculomotor behavior, and this may reflect the presence of individual differences in the awareness of observers' own oculomotor responses. As a matter of fact, recent work has shown how eye movements are very often not under volitional control, and even though observers may feel that they are following instructions and fixating on the center of the screen, saccadic responses might show otherwise (Clarke, Mahon, Irvine, \& Hunt, 2017; Mahon, Clarke, \& Hunt, 2018). Our second experiment intended to overcome the limitation of the first experimental design regarding the large variability between individual participants as well as to test the statistic reliability of analyses done in Experiment 1. We further explored the role of eye movements by checking whether the patterns of results remained consistent across two tasks differing in difficulty. A simple manipulation of the target shape proved to be very effective in increasing the need for eye movements, while preserving the main features of the cueing task.

In our experiments, we distinguished two main distributions of eye movements. The first one likely represents overt responses to the presentation of the central cues, before the target and distractor are displayed. The second distribution reflects eye movements made once the target and distractor have appeared on the screen. What do these event-related eye movements reveal about the processing of nonpredictive central cues? On the one hand, the examination of cue-elicited saccades confirmed that overt performance is susceptible to uninformative central cues, irrespective of whether the cue was social or not. Furthermore, saccade landing positions of target-elicited saccades were also comparable in the two cue conditions. When the task was easy in Experiment 1, saccadic eye movements landed closer to the target only in the valid condition. It seems that these eye movements were mainly executed to get a better look at the target to support target discrimination. This pattern changed when the task became more difficult, in the sense that saccades appeared to follow the nonpredictive central cues, irrespective of their validity. Critically, all of these measures of oculomotor behavior failed to reveal any differences between social and nonsocial cues. Our work shows that this basic form of social attention (i.e., attentional orienting in response to eyes) is similar to nonsocial orienting of attention in most oculomotor measures.

The only difference we found between social and nonsocial cues was in saccade latency, which depended distinctively on the type of cue. In this regard, significant differences in saccade latency may support the notion that eye-gaze cues are processed more rapidly than nonsocial cues and that gaze cues lead to more reflexive orienting of overt attention. It may be the case that this difference can be explained by low-level differences between the gaze cue and the arrow cue. The gaze cue was far more complex compared with the line drawing of the arrow. Yet despite the fact that the stimuli were so vastly different, our results showed that they influenced manual behavior in a very similar way. Previous studies that more systematically controlled for low-level differences of social and nonsocial stimuli, as well as included instructions about the type of eye movement to execute, also reported faster latencies in response to the gaze cue for both for correct saccades (Kuhn \& Kingstone, 2009) and erroneous saccades (Kuhn \& Benson, 2007). In an attempt to increase ecological validity of the social stimuli, we used a more realistic eye-gaze cue; regardless of stimulus-complexity differences, our data are in line with previous findings on saccade latency showing faster response to gaze than to arrows. Importantly, both cue-elicited and target-elicited eye movements uncovered this subtle difference between social and nonsocial cues, and this further shows that saccade latencies are immediately affected by the type of cue, independent of the presentation of the target or task.

Interestingly, this effect appears to be preserved also in overall RTs, providing evidence for a cue-dependent association between oculomotor and manual responses. While Experiment 1 highlighted a trend in this direction in the subgroup of individuals that relied more on eye movements, Experiment 2 confirmed this result in a bigger sample of participants. Irrespective of task difficulty, the gaze-cue advantage on latency and overall RT was present when we matched samples for eye movements. The fact that eye gaze has a greater impact on selection may find its roots in difference in biological relevance; while the eye gaze is important for humans to develop social interaction and joint attention (Corkum \& Moore, 1995), the arrow cue is typically regarded as nonbiologically relevant. Several experimental studies have tested this hypothesis comparing the social and nonsocial cues both in terms of their behavioral effects as well as the possible neural mechanisms underlying this form of reflexive orienting (Carlin \& Calder, 2013; Dalmaso, Galfano, Tarqui, Forti, \& Castelli, 2013; Friesen, Moore, \& Kingstone, 2005; Friesen et al., 2004; Galfano et al., 2012; Galfano et al., 2011; Hietanen, Leppänen, Nummenmaa, \& Astikainen, 2008; Hietanen, Nummenmaa, Nyman, Parkkola, \& Hämäläinen, 2006; Kingstone, Tipper, Ristic, \& Ngan, 2004; Langdon \& Smith, 2005; Ristic et al., 2002; Ristic et al., 2007). However, the debate is far from being settled. While several experimental and neuropsychological studies have provided evidence in favor of the idea that gaze-cue processing is more reflexive and less affected by voluntary control of attention, and that additionally these cues rely on diverse neural networks (Akiyama et al., 2006; Friesen et al., 2005; Friesen et al., 2004; Hietanen et al., 2008; Kingstone, Friesen, \& Gazzaniga, 2000; Ristic et al., 2002; Ristic \& Kingstone, 
2005; Ristic et al., 2007), others have found no evidence to suggest differences between the social and nonsocial cues (e.g., Galfano et al., 2012; Kuhn \& Benson, 2007; Kuhn \& Kingstone, 2009; Tipples, 2008).

While the present patterns of results on saccade latency and on manual overall response times support this distinction, they also suggest that this difference between gaze and arrow cues is apparent only in the presence of eye movements. As a matter of fact, no cue modulation on RT was found in the subgroup of participants that did not move the eyes in Experiment 1, but it was clearly present in Experiment 2, where all participants needed eye movements for the discrimination task. It may be possible that eye contact prompts observers to make eye movements more rapidly and to respond faster, thus highlighting the gaze-cue advantage on latency and overall RT. This effect may stem from a general alerting effect caused by the eyes (see also Kuhn \& Benson, 2007; Kuhn \& Kingstone, 2009), or it may represent a unique social feature of attentional orienting to social cues (Marotta, RománCaballero, \& Lupiáñez, 2018). Future research could focus on specifying in more detail in which contexts such facilitation emerges, and which are the possible factors driving this effect. Simplifying the qualitative features of the face or, more challenging, enriching the sensory characteristics of the nonsocial cue could facilitate the comparison between social and nonsocial cues in different situations. In our case, it could be argued that very different low-level features like saliency and size may have played a role in creating the advantage on overall RTs observed in the gaze-cue condition. Nonetheless, even if saccades were elicited faster in response to gaze cues, not only when the target was presented but also before that moment, it is important to note that the magnitude of the validity effect on RT was not affected by cue type.

While saccade latency did not vary as a function of validity, evidence for a strong link between attention and eye movements was found in the association between the validity effect on saccade landing positions and the cueing effect on RTs. Moreover, this distinction between saccade latency and landing position further suggests that not all aspects of overt selection are affected by the type of cue. Regardless of cue type, landing position of target-elicited initial eye movements in valid and invalid trials was tightly associated with the magnitude of the final validity effect on RTs. Taking into account that initial target-elicited saccades had their peak at around $200 \mathrm{~ms}$ after the target onset and responses occurred 400 $500 \mathrm{~ms}$ later, our data endorses the statement that nonpredictive cues elicit a form of orienting that is resistant to volition and persists over time. We confirmed this robust association also in a larger sample size and with bigger data sets at disposal (Experiment 2). Yet more research is required to verify the existence of a causal relationship between direction of initial eye movements and cueing effects on manual responses. As we did not directly manipulate oculomotor measures, any interpretation of the causal relationship between landing position and manual cueing effect remains premature, and more research is needed to test it. Nonetheless, the examination of eye movements in a spatial cueing paradigm revealed the presence of distinctive associations between diverse oculomotor components and manual RT. Our data demonstrate that saccade latency is cue dependent and that the gaze-cue facilitation seems to extend to the manual responses. Conversely, landing position is validity-dependent and is tightly associated with the magnitude of cueing effects on RT.

These findings have implications for our understanding of the relationship between attention and eye movements. Our study suggests that different components of eye movements seem to be differently associated with the observed final manual response; this shows that the relationship between attentional orienting and eye movements is not straightforward. Although interpretations of this distinction of oculomotor parameters are somewhat premature and further research is needed, they seem to suggest that the when and the where of eye movements have different properties and separate links to manual responses, at least when nonpredictive social and nonsocial cues are involved and compared with each other. Under this view, different components of saccadic eye movements may be independently associated with the final attentional performance on manual RTs. Future research should aim at verifying whether these relationships may be the sign of independent contributions of these saccade parameters, in terms of cause-effect, on motor programming and execution of manual responses.

In this study we explored overt selection by examining the latency and landing position of initial saccades. However, in future studies, other parameters may be evaluated to help expand the current results. For example, microsaccades (Engbert \& Kliegl, 2003; Hafed \& Clark, 2002; Valsecchi, Betta, \& Turatto, 2007) may be a good source of information to study the dynamics of attention of individuals characterized by a low rate of eye movements. Specifically, in our case, in Experiment 1, when the task was easy enough to be performed without involving any kind of saccades. One hypothesis that could be tested is that peak velocity and amplitude of microsaccades are affected by cue type just as saccades, showing a greater influence of the social cue on these parameters. However, since microsaccades are significantly affected by perceptual features of the stimuli (McCamy, Jazi, OteroMillan, Macknik, \& Martinez-Conde, 2013), a comparison between social and nonsocial cues would require a more systematic control of size and complexity in order to closely match the perceptual features of the two central cues.

Our study suggests that it is possible to combine the analysis of the two components of orienting in one task, and that this approach can help disclose links between overt selection and manual response. We pointed out how the eye gaze has an impact on latency that appears to persist until the final manual response. We also found a very strong association between 
validity effects on initial saccades and the magnitude of the final validity effect. Our findings clearly point out the benefit of concurrently monitoring of eye movements in spatial cueing paradigms without explicit task instructions to make eye movements. Our results support the idea that the systematic analysis of eye movements can help map the time course and dynamics of cueing over time. This approach, together with a more precise characterization of the context in which orienting of attention occurs, could shed light on the debate regarding similarities and differences of social and nonsocial cues.

Acknowledgements This research was supported by the Autonomous Province of Trento (Italy), call Grandi Progetti 2012, project Characterizing and Improving Brain Mechanisms of AttentionATTEND. The authors report no conflict of interests. Finally, we thank Elisa Crestale for having helped in the data collection of Experiment 2.

Publisher's note Springer Nature remains neutral with regard to jurisdictional claims in published maps and institutional affiliations.

\section{References}

Akiyama, T., Kato, M., Muramatsu, T., Saito, F., Umeda, S., \& Kashima, H. (2006). Gaze but not arrows: A dissociative impairment after right superior temporal gyrus damage. Neuropsychologia, 44(10), 1804-1810. https://doi.org/10.1016/j.neuropsychologia.2006.03. 007

Anderson, S. F., Kelley, K., \& Maxwell, S. E. (2017). Sample-size planning for more accurate statistical power: A method adjusting sample effect sizes for publication bias and uncertainty. Psychological Science, 28(11), 1547-1562. https://doi.org/10.1177/ 0956797617723724

Bertelson, P. (1967). The time course of preparation*. Quarterly Journal of Experimental Psychology, 19(3), 272-279. https://doi.org/10. 1080/14640746708400102

Blair, C., Capozzi, F., \& Ristic, J. (2017). Where is your attention? Assessing individual instances of covert attentional orienting in response to gaze and arrow cues. Vision, 1(3), 19. https://doi.org/10. 3390/vision 1030019

Carlin, J. D., \& Calder, A. J. (2013). The neural basis of eye gaze processing. Current Opinion in Neurobiology, 23(3), 450-455. https:// doi.org/10.1016/j.conb.2012.11.014

Clarke, A. D. F., Mahon, A., Irvine, A., \& Hunt, A. R. (2017). People are unable to recognize or report on their own eye movements. Quarterly Journal of Experimental Psychology, 70(11). https://doi. org/10.1080/17470218.2016.1231208

Corbetta, M., \& Shulman, G. L. (2002). Control of goal-directed and stimulus-driven attention in the brain. Nature Reviews Neuroscience, 3(3), 201-215. https://doi.org/10.1038/nrn755

Corkum, V., \& Moore, C. (1995). Development of joint visual attention in infants. In C. Moore \& P. J. Dunham (Series), Joint attention: Its origins and role in development (pp. 61-83). Hillsdale: Erlbaum.

Dalmaijer, E. S., Mathôt, S., \& Van der Stigchel, S. (2014). PyGaze: An open-source, cross-platform toolbox for minimal-effort programming of eyetracking experiments. Behavior Research Methods, 46(4), 913-921. https://doi.org/10.3758/s13428-013-0422-2

Dalmaso, M., Castelli, L., Franchetti, L., Carli, L., Todisco, P., Palomba, D., \& Galfano, G. (2015). Altered orienting of attention in anorexia nervosa. Psychiatry Research, 229(1), 318-325. https://doi.org/10. 1016/j.psychres.2015.06.044
Dalmaso, M., Galfano, G., Tarqui, L., Forti, B., \& Castelli, L. (2013). Is social attention impaired in schizophrenia? Gaze, but not pointing gestures, is associated with spatial attention deficits. Neuropsychology, 27(5), 608-613. https://doi.org/10.1037/ a0033518

Driver, J., Davis, G., Ricciardelli, P., Kidd, P., Maxwell, E., \& BaronCohen, S. (1999). Gaze perception triggers reflexive visuospatial orienting. Visual Cognition, 6(5), 509-540. https://doi.org/10. 1080/135062899394920

Egeth, H. E., \& Yantis, S. (1997). Visual attention: Control, representation, and time course. Annual Review of Psychology, 48(1), 269297. https://doi.org/10.1146/annurev.psych.48.1.269

Engbert, R., \& Kliegl, R. (2003). Microsaccades uncover the orientation of covert attention. Vision Research, 43(9), 1035-1045. https://doi. org/10.1016/S0042-6989(03)00084-1

Friesen, C. K., \& Kingstone, A. (1998). The eyes have it! Reflexive orienting is triggered by nonpredictive gaze. Psychonomic Bulletin \& Review, 5(3), 490-495. https://doi.org/10.3758/BF03208827

Friesen, C. K., Moore, C., \& Kingstone, A. (2005). Does gaze direction really trigger a reflexive shift of spatial attention? Brain and Cognition, 57(1), 66-69. https://doi.org/10.1016/j.bandc.2004.08. 025

Friesen, C. K., Ristic, J., \& Kingstone, A. (2004). Attentional effects of counterpredictive gaze and arrow cues. Journal of Experimental Psychology: Human Perception and Performance, 30(2), 319329. https://doi.org/10.1037/0096-1523.30.2.319

Frischen, A., Bayliss, A. P., \& Tipper, S. P. (2007). Gaze cueing of attention: Visual attention, social cognition, and individual differences. Psychological Bulletin, 133(4), 694-724. https://doi.org/10.1037/ 0033-2909.133.4.694

Galfano, G., Dalmaso, M., Marzoli, D., Pavan, G., Coricelli, C., \& Castelli, L. (2012). Eye gaze cannot be ignored (but neither can arrows). Quarterly Journal of Experimental Psychology, 65(10), 1895-1910. https://doi.org/10.1080/17470218.2012.663765

Galfano, G., Sarlo, M., Sassi, F., Munafò, M., Fuentes, L. J., \& Umiltà, C. (2011). Reorienting of spatial attention in gaze cuing is reflected in N2pc. Social Neuroscience, 6(3), 257-269. https://doi.org/10.1080/ 17470919.2010.515722

Gibson, B. S., \& Bryant, T. A. (2005). Variation in cue duration reveals top-down modulation of involuntary orienting to uninformative symbolic cues. Perception \& Psychophysics, 67(5), 749-758. https://doi.org/10.3758/BF03193530

Hafed, Z. M., \& Clark, J. J. (2002). Microsaccades as an overt measure of covert attention shifts. Vision Research, 42(22), 2533-2545. https:// doi.org/10.1016/S0042-6989(02)00263-8

Heimler, B., van Zoest, W., Baruffaldi, F., Rinaldi, P., Caselli, M. C., \& Pavani, F. (2015). Attentional orienting to social and nonsocial cues in early deaf adults. Journal of Experimental Psychology: Human Perception and Performance, 41(6), 1758-1771. https://doi.org/10. 1037/xhp0000099

Hietanen, J. K. (1999). Does your gaze direction and head orientation shift my visual attention? NeuroReport, 10(16), 3443.

Hietanen, J. K., Leppänen, J. M., Nummenmaa, L., \& Astikainen, P. (2008). Visuospatial attention shifts by gaze and arrow cues: An ERP study. Brain Research, 1215, 123-136. https://doi.org/10. 1016/j.brainres.2008.03.091

Hietanen, J. K., Nummenmaa, L., Nyman, M. J., Parkkola, R., \& Hämäläinen, H. (2006). Automatic attention orienting by social and symbolic cues activates different neural networks: An fMRI study. NeuroImage, 33(1), 406-413. https://doi.org/10.1016/j. neuroimage.2006.06.048

Hommel, B., Pratt, J., Colzato, L., \& Godijn, R. (2001). Symbolic control of visual attention. Psychological Science, 12(5), 360-365. https:// doi.org/10.1111/1467-9280.00367

Hood, B. M., Willen, J. D., \& Driver, J. (1998). Adult's eyes trigger shifts of visual attention in human infants. Infant Behavior and 
Development, 21, 466. https://doi.org/10.1016/S0163-6383(98) 91679-4

Jonides, J. (1981). Voluntary versus automatic control over the mind's eye's movements. In J. B. Long \& A. D. Baddeley (Eds.), Attention and performance IX (pp. 187-203). Hillsdale: Erlbaum.

Kingstone, A., Friesen, C. K., \& Gazzaniga, M. S. (2000). Reflexive joint attention depends on lateralized cortical connections. Psychological Science, 11(2), 159-166. https://doi.org/10.1111/1467-9280.00232

Kingstone, A., Tipper, C., Ristic, J., \& Ngan, E. (2004). The eyes have it!: An fMRI investigation. Brain and Cognition, 55(2), 269-271. https://doi.org/10.1016/j.bandc.2004.02.037

Kröse, B. J. A., \& Julesz, B. (1989). The control and speed of shifts of attention. Vision Research, 29(11), 1607-1619. https://doi.org/10. 1016/0042-6989(89)90142-9

Kuhn, G., \& Benson, V. (2007). The influence of eye-gaze and arrow pointing distractor cues on voluntary eye movements. Perception \& Psychophysics, 69(6), 966-971. https://doi.org/10.3758/ BF03193934

Kuhn, G., Benson, V., Fletcher-Watson, S., Kovshoff, H., McCormick, C. A., Kirkby, J., \& Leekam, S. R. (2009a). Eye movements affirm: automatic overt gaze and arrow cueing for typical adults and adults with autism spectrum disorder. Experimental Brain Research, 201(2), 155-165. https://doi.org/10.1007/s00221-009-2019-7

Kuhn, G., \& Kingstone, A. (2009). Look away! Eyes and arrows engage oculomotor responses automatically. Attention, Perception \& Psychophysics, 71(2), 314-327. https://doi.org/10.3758/APP.71.2. 314

Kuhn, G., Tatler, B. W., \& Cole, G. G. (2009b). You look where I look! Effect of gaze cues on overt and covert attention in misdirection. Visual Cognition, 17(6/7), 925-944. https://doi.org/10.1080/ 13506280902826775

Kuhn, G., Tewson, L., Morpurgo, L., Freebody, S. F., Musil, A. S., \& Leekam, S. R. (2011). Developmental changes in the control of saccadic eye movements in response to directional eye gaze and arrows. Quarterly Journal of Experimental Psychology, 64(10), 1919-1929. https://doi.org/10.1080/17470218.2011.592592

Langdon, R., \& Smith, P. (2005). Spatial cueing by social versus nonsocial directional signals. Visual Cognition, 12(8), 1497-1527. https:// doi.org/10.1080/13506280444000805

Langton, S. R. H., \& Bruce, V. (1999). Reflexive visual orienting in response to the social attention of others. Visual Cognition, 6(5), 541-567. https://doi.org/10.1080/135062899394939

Mahon, A., Clarke, A. D. F., \& Hunt, A. R. (2018). The role of attention in eye-movement awareness. Attention, Perception, \& Psychophysics, 80(7), 1691-1704. https://doi.org/10.3758/s13414018-1553-4

Mansfield, E., Farroni, T., \& Johnson, M. (2003). Does gaze perception facilitate overt orienting? Visual Cognition, 10(1), 7-14. https://doi. org $/ 10.1080 / 713756671$

Marotta, A., Román-Caballero, R., \& Lupiáñez, J. (2018). Arrows don't look at you: Qualitatively different attentional mechanisms triggered by gaze and arrows. Psychonomic Bulletin \& Review. https://doi.org/ 10.3758/s13423-018-1457-2

Mathôt, S., Schreij, D., \& Theeuwes, J. (2012). OpenSesame: An opensource, graphical experiment builder for the social sciences. Behavior Research Methods, 44(2), 314-324. https://doi.org/10. 3758/s13428-011-0168-7

McCamy, M. B., Jazi, A. N., Otero-Millan, J., Macknik, S. L., \& Martinez-Conde, S. (2013). The effects of fixation target size and luminance on microsaccades and square-wave jerks. PeerJ, 1 , e9. https://doi.org/10.7717/peerj.9

Müller, H. J., \& Humphreys, G. W. (1991). Luminance-increment detection: Capacity-limited or not? Journal of Experimental Psychology: Human Perception and Performance, 17(1), 107-124. https://doi. org/10.1037/0096-1523.17.1.107
Müller, H. J., \& Rabbitt, P. M. (1989). Reflexive and voluntary orienting of visual attention: Time course of activation and resistance to interruption. Journal of Experimental Psychology: Human Perception and Performance, 15(2), 315-330. https://doi.org/10.1037/00961523.15.2.315

O'Brien, F., \& Cousineau, D. (2014). Representing Error bars in withinsubject designs in typical software packages. The Quantitative Methods for Psychology, 10(1), 56-67. https://doi.org/10.20982/ tqmp.10.1.p056

Oosterhof, N. N., \& Todorov, A. (2008). The functional basis of face evaluation. Proceedings of the National Academy of Sciences, 105(32), 11087-11092. https://doi.org/10.1073/pnas.0805664105

Peirce, J. W. (2007). PsychoPy-Psychophysics software in Python. Journal of Neuroscience Methods, 162(1/2), 8-13. https://doi.org/ 10.1016/j.jneumeth.2006.11.017

Peirce, J. W. (2009). Generating stimuli for neuroscience using PsychoPy. Frontiers in Neuroinformatics, 2. https://doi.org/10.3389/neuro.11. 010.2008

Posner, M. I. (1978). Chronometric explorations of mind. Oxford: Erlbaum.

Posner, M. I. (1980). Orienting of attention. Quarterly Journal of Experimental Psychology, 32(1), 3-25. https://doi.org/10.1080/ 00335558008248231

Ricciardelli, P., Bricolo, E., Aglioti, S. M., \& Chelazzi, L. (2002). My eyes want to look where your eyes are looking: Exploring the tendency to imitate another individual's gaze. NeuroReport, 13(17), 2259.

Ristic, J., Friesen, C. K., \& Kingstone, A. (2002). Are eyes special? It depends on how you look at it. Psychonomic Bulletin \& Review, 9(3), 507-513. https://doi.org/10.3758/BF03196306

Ristic, J., \& Kingstone, A. (2005). Taking control of reflexive social attention. Cognition, 94(3), B55-B65. https://doi.org/10.1016/j. cognition.2004.04.005

Ristic, J., \& Kingstone, A. (2009). Rethinking attentional development: Reflexive and volitional orienting in children and adults. Developmental Science, 12(2), 289-296. https://doi.org/10.1111/j. 1467-7687.2008.00756.x

Ristic, J., Wright, A., \& Kingstone, A. (2007). Attentional control and reflexive orienting to gaze and arrow cues. Psychonomic Bulletin \& Review, 14(5), 964-969. https://doi.org/10.3758/BF03194129

Stevens, S. A., West, G. L., Al-Aidroos, N., Weger, U. W., \& Pratt, J. (2008). Testing whether gaze cues and arrow cues produce reflexive or volitional shifts of attention. Psychonomic Bulletin \& Review, 15(6), 1148-1153. https://doi.org/10.3758/PBR.15.6.1148

Tipples, J. (2002). Eye gaze is not unique: Automatic orienting in response to uninformative arrows. Psychonomic Bulletin \& Review, 9(2), 314-318. https://doi.org/10.3758/BF03196287

Tipples, J. (2008). Orienting to counterpredictive gaze and arrow cues. Perception \& Psychophysics, 70(1), 77-87. https://doi.org/10.3758/ PP.70.1.77

Valsecchi, M., Betta, E., \& Turatto, M. (2007). Visual oddballs induce prolonged microsaccadic inhibition. Experimental Brain Research, 177(2), 196-208. https://doi.org/10.1007/s00221-006-0665-6

Vecera, S. P., \& Rizzo, M. (2004). What are you looking at?: Impaired 'social attention' following frontal-lobe damage. Neuropsychologia, 42(12), 1657-1665. https://doi.org/10.1016/j.neuropsychologia. 2004.04.009

Vecera, S. P., \& Rizzo, M. (2006). Eye gaze does not produce reflexive shifts of attention: Evidence from frontal-lobe damage. Neuropsychologia, 44(1), 150-159. https://doi.org/10.1016/j. neuropsychologia.2005.04.010

Wright, R. D., \& Ward, L. M. (2008). Orienting of attention. Oxford: Oxford University Press. 